\title{
Cardiovascular adverse events in patients with chronic lymphocytic leukemia receiving acalabrutinib monotherapy: pooled analysis of 762 patients
}

\begin{abstract}
Jennifer R. Brown, ${ }^{1}$ John C. Byrd, ${ }^{2}$ Paolo Ghia, ${ }^{3}$ Jeff P. Sharman, ${ }^{4}$ Peter Hillmen, ${ }^{5}$ Deborah M. Stephens, ${ }^{6}$ Clare Sun, ${ }^{7}$ Wojciech Jurczak, ${ }^{8}$ John M. Pagel, ${ }^{9}$ Alessandra Ferrajoli, ${ }^{10}$ Priti Patel, ${ }^{11}$ Lin Tao, ${ }^{11}$ Nataliya Kuptsova-Clarkson, ${ }^{12}$ Javid Moslehi ${ }^{13}$ and Richard R. Furman ${ }^{14}$

${ }^{1}$ Dana-Farber Cancer Institute, Boston, MA, USA; ${ }^{2}$ The Ohio State University Comprehensive Cancer Center, Columbus, OH, USA; ${ }^{3}$ Università Vita-Salute San Raffaele and IRCCS Ospedale San Raffaele, Milano, Italy; ${ }^{4}$ Willamette Valley Cancer Institute/US Oncology, Eugene, OR, USA; ${ }^{5}$ St. James's University Hospital, Leeds, UK; ${ }^{6}$ University of Utah Huntsman Cancer Institute, Salt Lake City, UT, USA; ${ }^{7}$ National Heart, Lung, and Blood Institute, Bethesda, MD, USA; ${ }^{8}$ Maria Sklodowska-Curie National Research Institute of Oncology, Krakow, Poland; ${ }^{9}$ Swedish Cancer Institute, Seattle, WA, USA; ${ }^{10}$ University of Texas MD Anderson Cancer Center, Houston, TX, USA; ${ }^{11}$ AstraZeneca, South San Francisco, CA, USA; ${ }^{12}$ AstraZeneca, Gaithersburg, MD, USA; ${ }^{13}$ Section of Cardio-Oncology \& Immunology, Division of Cardiology and the Cardiovascular Research Institute, University of California San Francisco, San Francisco, CA, USA and ${ }^{14}$ Weill Cornell Medicine, New York Presbyterian Hospital, New York, NY, USA
\end{abstract}

\author{
Correspondence: \\ Jennifer R. Brown \\ jennifer_brown@dfci.harvard.edu \\ Received: $\quad$ April 6, 2021. \\ Accepted: $\quad$ September 21, 2021 \\ Prepublished: September 30,2021 \\ https://doi.org/10.3324/haematol.2021.278901 \\ C2022 Ferrata Storti Foundation \\ Haematologica material is published under a \\ CC BY-NC license (c) (1) \&
}

\begin{abstract}
Cardiovascular (CV) toxicities of the Bruton tyrosine kinase (BTK) inhibitor ibrutinib may limit use of this effective therapy in patients with chronic lymphocytic leukemia (CLL). Acalabrutinib is a second-generation BTK inhibitor with greater BTK selectivity. This analysis characterizes pooled CV adverse events (AE) data in patients with CLL who received acalabrutinib monotherapy in clinical trials (clinicaltrials gov. Identifier: NCT02029443, NCT02475681, NCT02970318 and NCT02337829). Acalabrutinib was given orally at total daily doses of 100-400 mg, later switched to $100 \mathrm{mg}$ twice daily, and continued until disease progression or toxicity. Data from 762 patients (median age: 67 years [range, 32-89]; median follow-up: 25.9 months [range, 0-58.5]) were analyzed. Cardiac AE of any grade were reported in 129 patients (17\%; grade $\geq 3, n=37[5 \%]$ ) and led to treatment discontinuation in seven patients $(1 \%)$. The most common any-grade cardiac AE were atrial fibrillation/flutter (5\%), palpitations (3\%), and tachycardia (2\%). Overall, $91 \%$ of patients with cardiac AE had CV risk factors before acalabrutinib treatment. Among 38 patients with atrial fibrillation/flutter events, seven (18\%) had prior history of arrhythmia or atrial fibrillation/flutter. Hypertension AE were reported in 67 patients (9\%), 43 (64\%) of whom had a preexisting history of hypertension; no patients discontinued treatment due to hypertension. No sudden cardiac deaths were reported. Overall, these data demonstrate a low incidence of new-onset cardiac AE with acalabrutinib in patients with CLL. Findings from the head-to-head, randomized trial of ibrutinib and acalabrutinib in patients with highrisk CLL (clinicaltrials gov. Identifier: NCT02477696) prospectively assess differences in CV toxicity between the two agents.
\end{abstract}

\section{Introduction}

Bruton tyrosine kinase (BTK) inhibitors, such as ibrutinib and acalabrutinib, are preferred treatment regimens for chronic lymphocytic leukemia (CLL). BTK is involved in downstream amplification of $\mathrm{B}$-cell receptor signaling and is a key anticancer target for CLL and other B-cell malignancies such as mantle cell lymphoma and Waldenström's macroglobulinemia. ${ }^{2-6}$

Ibrutinib was the first BTK inhibitor approved for the treatment of CLL. ${ }^{2}$ In addition to irreversibly binding to the cysteine residue (C481) and blocking the adenosine triphosphate (ATP)-binding pocket of BTK, $, 7,8$ ibrutinib binds to analogous cysteine residues in other kinases such as interleukin-2-inducible T-cell kinase (ITK), tyrosine kinase expressed in hepatocellular carcinoma (TEC), epidermal growth factor receptor (EGFR), and T-cell X chromosome kinase (TXK) as well as to several Src family kinases. ${ }^{9-11}$ Cardiovascular (CV) toxicities, particularly hypertension and atrial fibrillation, have been associated with ibrutinib, which may be attributed in part to off-target kinase inhibition. ${ }^{12-15}$ In the RESONATE study in patients with pre- 
viously treated CLL, atrial fibrillation of any grade was reported in $5 \%$ of patients receiving ibrutinib monotherapy at a median follow-up of 9.4 months, which increased to $11 \%$ with 44 months of follow-up; in the RESONATE-2 study in patients with treatment-naïve CLL, any-grade atrial fibrillation was reported in $6 \%$ and $11 \%$ of patients at median follow-up durations of 18.4 and 60 months, respectively. ${ }^{16-19}$ Additionally, in the CLL-12 trial in patients with asymptomatic, treatment-naïve, early stage CLL, any-grade atrial fibrillation was reported in $18 \%$ of patients receiving ibrutinib versus $7 \%$ of patients on placebo after a median follow-up of 31 months, with grade 3 to 4 events reported in $6 \%$ and $2 \%$ of patients, respectively. ${ }^{20}$ With longer follow-up in the phase Ib/II PCYC-1102 ibrutinib trial, atrial fibrillation of grade 3 or higher was reported in $10 \%$ of patients with relapsed/refractory CLL at a median of 6.8 years of follow-up and in $6 \%$ of patients with treatment-naive CLL at a median of 7.3 years of follow-up.19 A review of 16 ibrutinib clinical studies found the rate of any-grade atrial fibrillation (4-16\%) to be higher than that in a general population (1-2\%). ${ }^{21}$ In a singlecenter, retrospective analysis in ibrutinib-treated patients $(n=562)$, new or worsening hypertension was associated with an increased risk of atrial fibrillation..$^{14,22}$

Acalabrutinib, a second-generation, potent, highly selective, covalent BTK inhibitor, was approved for the treatment of CLL based on the results of two randomized, controlled phase III studies, ELEVATE-TN and ASCEND., ${ }^{3,23,24}$ Compared with ibrutinib, acalabrutinib has greater selectivity for BTK in vitro, 7,9 which has been hypothesized to explain differences in the tolerability profiles of the two agents. ${ }^{6,9,16,25}$ At median follow-up duration of 16.1 months in the ASCEND studies, the incidence of atrial fibrillation of any grade was $5 \%$ in the acalabrutinib monotherapy arm compared with $3 \%$ in the comparator arm. ${ }^{23,24}$ Similarly, at a median follow-up of 28.3 months in the ELEVATE-TN study, the incidence of any-grade atrial fibrillation was $4 \%$ in the acalabrutinib monotherapy arm versus $1 \%$ in the comparator arm. ${ }^{23}$

In order to further explore cardiac and hypertension-related effects of acalabrutinib in patients with CLL, we conducted a retrospective pooled analysis of data from all clinical studies of acalabrutinib (from phase I to III) in CLL containing an acalabrutinib monotherapy arm and characterized CV adverse events (AE).

\section{Methods}

Data were from a pooled population of patients with CLL treated with at least one dose of acalabrutinib monotherapy in all sponsored clinical trials containing an acalabrutinib monotherapy arm (Table 1).9,23,24,26-29 Because of the inclusion of a phase I study and an initial phase II study, acalabrutinib was given orally at total daily doses of 100$400 \mathrm{mg}$ (although the majority of patients in this analysis [78\%] received $100 \mathrm{mg}$ twice daily [BID]). Treatment continued until progressive disease or toxicity. Each study's protocol was approved by an Institutional Review Board and each study was conducted in accordance with the general principles set forth in the International Conference on Harmonization Guidelines for Good Clinical Practice and the Declaration of Helsinki, and in accordance with all applicable legal and regulatory requirements. All participants gave written informed consent.

AE were coded using Medical Dictionary for Regulatory Activities v21.1. Severity was graded according to National Cancer Institute Common Terminology Criteria for Adverse Events (CTCAE) v4.03. The protocols defined AE as those occurring or worsening on or after the first dose of acalabrutinib, through the treatment phase, and within 30 days of the last dose. For this analysis, cardiac AE were those categorized under the system organ class "cardiac disorders"; hypertension AE, which were considered an AE of clinical interest, included the following preferred terms: hypertension, blood pressure increased, essential hyper-

Table 1. Study summaries.

\begin{tabular}{|c|c|c|}
\hline Study name & Study description & Number of patients ${ }^{a}$ \\
\hline ACE-CL-001 (NCT02029443) & $\mathrm{Ph} \mathrm{I} / \mathrm{II}$ study of acalabrutinib in patients with CLL & 301; TN/RR: 112/189 \\
\hline $\begin{array}{l}\text { ACE-CL-007 (NCT02475681; } \\
\text { ELEVATE-TN) }^{\mathrm{b}}\end{array}$ & Ph III study of acalabrutinib $\pm \mathrm{O}$ vs $\mathrm{C}+\mathrm{O}$ in TN CLL & 224; all TN \\
\hline ACE-CL-309 (NCT02970318; ASCEND) & Ph III study of acalabrutinib vs IdR or BR in RR CLL & 189; all RR \\
\hline 15-H-0016 (NCT02337829) & $\begin{array}{l}\text { Ph II study of acalabrutinib in patients with RR or TN } \\
\text { with del(17p) CLL }\end{array}$ & 48; TN/RR: 16/32 \\
\hline
\end{tabular}
nutuzumab; PD: progressive disease; Ph: phase; PO: orally; RR: relapsed/refractory; TN: treatment-naïve. 
tension, hypertensive crisis, malignant hypertension, hypertensive heart disease, and orthostatic hypertension.

Assessments of cardiac $A E$ and hypertension $A E$ included incidence, rate per patient exposure years, time to onset, incidence of events leading to dose delays and discontinuations, and event management. Event duration was also assessed for overall cardiac $A E$ and atrial fibrillation/flutter events. Cardiac AE severity and resolution of grade $\geq 3$ cardiac $A E$ also were assessed. Incidence of cardiac $A E$ in the first 6 months was assessed based on a predominance of atrial fibrillation events during this time period in published reports with ibrutinib.13 CV and hypertension risk factors, based on patient medical histories, were also assessed (methodology described in the Online Supplementary Appendix). Prior history of arrhythmia, atrial fibrillation, and atrial flutter events was assessed in patients who experienced atrial fibrillation events, and prior history of hypertension was assessed in patients who experienced hypertension events. Occurrence of major hemorrhage events (defined as any hemorrhagic event that was serious or grade $\geq 3$ in severity, or any central nervous system hemorrhage) after receipt of anticoagulant therapy on study was assessed.

Atrial fibrillation risk scores (range, 0-7) were computed in patients without a prior history of atrial fibrillation using the Shanafelt predictive model, calculated as the sum of the risk values assigned to the specific factors independently associated with atrial fibrillation: older age (2 points for age 65-74 years; 3 points for age $\geq 75$ years), male sex ( 1 point), valvular heart disease ( 2 points), and hypertension (1 point). ${ }^{13,30,31}$

\section{Statistical analysis}

Data were summarized using descriptive statistics. Time to first cardiac $A E$ overall, atrial fibrillation/flutter $A E$, and hypertension AE were analyzed using the Kaplan-Meier method. Incidence of atrial fibrillation was analyzed by age group ( $<65$ years, 65 to $<75$ years, $\geq 75$ years) and Shanafelt risk category $(0-1,2-3,4$, and $\geq 5)$. The incidence of cardiac AE also was compared between the pooled acalabrutinib monotherapy arms and pooled comparator arms from the randomized, controlled, phase III trials (ASCEND and ELEVATE-TN) included in this analysis; comparator treatments included investigator's choice of idelalisib plus rituximab or bendamustine plus rituximab (ASCEND) and obinutuzumab plus chlorambucil (ELEVATE-TN). Statistical comparison of cardiac AE incidence between acalabrutinib monotherapy and comparator arms was performed using the Chi-squared test.

\section{Results}

\section{Patients}

In total, 762 patients who received acalabrutinib monotherapy across four clinical trials were included in this analysis
(Table 1). Patient demographics and baseline characteristics were similar among the total acalabrutinib monotherapy CLL population and the subgroup of patients who experienced a cardiac AE (Table 2). At data cut-off, the median duration of follow-up was 25.9 month (range, $0.0-58.5 ; n=761$ ) and the median duration of acalabrutinib exposure was 24.9 months (range, 0.0-58.5; $n=760$ ). Five hundred fifty-three patients (73\%) remained on acalabrutinib at data cut-off. A total of 208 patients (27\%) discontinued acalabrutinib treatment; the most common reasons were progressive disease $(n=82 ; 11 \%)$ and $A E(n=70 ; 9 \%)$. When comparing the treatment-naïve $(n=352)$ and relapsed/refractory $(n=410)$ populations, $68(19 \%)$ and 140 (34\%) discontinued acalabrutinib treatment, respectively; the most common reasons were progressive disease $(n=19[5 \%]$ and $n=63$ [15\%], respectively) and $A E(n=27$ [8\%] and $n=43$ [10\%], respectively). Most patients (78\%; $\mathrm{n}=596 / 762)$ received only the $100 \mathrm{mg}$ BID dose of acalabrutinib; 166 patients (22\%) received a different initial acalabrutinib dose (55 received doses greater than $100 \mathrm{mg}$ BID [I.e., 200 $\mathrm{mg}$ BID, $250 \mathrm{mg}$ daily [QD], and $400 \mathrm{mg} Q \mathrm{QD}])$. Most of the patients who received an initial dose of acalabrutinib that differed from the standard dose of $100 \mathrm{mg}$ BID later switched to $100 \mathrm{mg} \operatorname{BID}(\mathrm{n}=106$ [64\%]). Patient demographics and baseline characteristics were similar in the pooled acalabrutinib monotherapy and comparator arms from ASCEND and ELEVATE-TN (Online Supplementary Table S1).

\section{Safety}

Cardiac adverse events

Across studies and exposure durations, there were 199 cardiac AE of any grade reported among 129 ( $n=58$ with treatment naïve CLL; $n=71$ with relapsed/refractory CLL) of 762 patients (17\%) (Table 3). Among the 55 patients who received initial acalabrutinib doses greater than $100 \mathrm{mg}$ BID, 12 (22\%) experienced cardiac AE, whereas 84 (14\%) reported cardiac AE among the 596 who had taken acalabrutinib only at the $100 \mathrm{mg}$ BID dose. In the overall population of 762 patients, the most common cardiac AE of any grade (incidence $\geq 2 \%$ ) were atrial fibrillation ( $4 \%[n=34]$; grade $\geq 3,1 \%$ [n=10]), palpitations ( $3 \%[n=23]$; grade $\geq 3,0)$, and tachycardia ( $2 \%[n=17]$; grade $\geq 3,0$ ). The median time to onset of cardiac AE was 10.1 months (range: $0.1-49.7)$ (Figure 1A). Overall, $7 \%(n=53 / 760)$ of patients reported any-grade cardiac $A E$ in the first 6 months on acalabrutinib, $3 \%(n=18 / 653)>6$ to 12 months after treatment initiation, and $8 \%(n=46 / 585)>12$ to 24 months after starting treatment. Cardiac AE led to acalabrutinib discontinuation in seven patients (1\%), six of whom discontinued due to grade $\geq 3$ cardiac events (described below) and one patient who discontinued due to grade 2 atrial fibrillation. Comparatively, 91\% ( $n=117 / 129)$ of patients with cardiac AE versus $80 \%(n=503 / 633)$ without cardiac AE had at least one $\mathrm{CV}$ risk factor before starting acalabrutinib. The most prevalent $\mathrm{CV}$ risk factors (defined in the Online Supplementary Appendix) for patients with versus 
Table 2. Patient demographics and baseline characteristics.

\begin{tabular}{|c|c|c|}
\hline Demographic/baseline characteristic & All patients $(\mathrm{N}=762)$ & $\begin{array}{c}\text { Patients with cardiac } \\
\text { events }(N=129)\end{array}$ \\
\hline Age, median (range), years & $67.0(32-89)$ & $69.0(42-84)$ \\
\hline Male, n (\%) & $508(66.7)$ & $83(64.3)$ \\
\hline \multicolumn{3}{|l|}{ Race, n (\%) } \\
\hline White & $698(91.6)$ & $113(87.6)$ \\
\hline Black or African American & $26(3.4)$ & $9(7.0)$ \\
\hline Asian & $14(1.8)$ & $3(2.3)$ \\
\hline American Indian or Alaska Native & $1(0.1)$ & 0 \\
\hline Native Hawaiian or Other Pacific Islander & $1(0.1)$ & 0 \\
\hline Other & $11(1.4)$ & $4(3.1)$ \\
\hline Missing & $11(1.4)$ & 0 \\
\hline BMI $\left(\mathrm{kg} / \mathrm{m}^{2}\right)$, median (range) & $26.7(16-49)^{a}$ & $27.1(18-47)^{b}$ \\
\hline \multicolumn{3}{|l|}{ ECOG PS score, n (\%) } \\
\hline 0 & $292(38.3)$ & $41(31.8)$ \\
\hline 1 & $415(54.5)$ & $81(62.8)$ \\
\hline 2 & $54(7.1)$ & $7(5.4)$ \\
\hline 3 & $1(0.1)$ & 0 \\
\hline Number of prior regimens, median (range) & $1(0-13)$ & $1(0-10)$ \\
\hline Treatment-naïve disease, n (\%) & $352(46.2)$ & $58(45.0)$ \\
\hline Relapsed/refractory disease, $\mathrm{n}(\%)$ & $410(53.8)$ & $71(55.0)$ \\
\hline
\end{tabular}

${ }^{a} n=746 .{ }^{b} n=126$. BMI: body mass index; ECOG PS: Eastern Cooperative Oncology Group performance status.

without cardiac AE were hypertension (65\% [n=84] vs. $53 \%$ [n=333], respectively), diabetes mellitus (19\% [n=24] vs. $12 \%[n=79])$, hyperlipidemia (16\% [n=21] vs. $11 \%$ [n=67]), hypothyroidism (16\% [n=21] vs. 12\% [n=73]), hypercholesterolemia (14\% [n=18] vs. $16 \%$ [ $n=104])$, and atrial fibrillation $(12 \%[n=16]$ vs. $6 \%[n=40])$. The most common ( $\geq 15 \%)$ types of concomitant medications reported among patients who experienced cardiac events were antithrombotic agents $(n=36[28 \%])$ and beta-blocking agents $(n=30$ [23\%]) (Online Supplementary Table S2); 16 patients (12\%) received both antithrombotic and beta-blocking agents, and a total of 17 patients (13\%) received aspirin (13 [10\%] received aspirin along with other antithrombotic agents and 4 [3\%] received aspirin without additional antithrombotic agents).

Fifty-one grade $\geq 3$ cardiac AE were reported among 37 patients (5\%). Overall, nine of 760 patients (1\%) experienced 13 grade $\geq 3$ cardiac AE (representing $25 \%$ of total grade $\geq 3$ events) during the first 6 months on acalabrutinib (Online Supplementary Table S3); six of 653 (1\%) and 15 of 585 patients (3\%) experienced grade $\geq 3$ cardiac $A E>6$ to 12 months and $>12$ to 24 months after starting acalabrutinib, respectively. Among the 37 patients with grade $\geq 3$ cardiac events, 12 patients experienced a grade 4 cardiac event and two patients experienced a grade 5 cardiac event. The grade $5 \mathrm{AE}$ included acute myocardial infarction in one patient with preexisting hypertension, type 2 diabetes mellitus, CV disorder, and chronic kidney disease; and congestive cardiac failure in another patient with a history of congestive cardiac failure, aortic stenosis, and atrial fibrillation. Among the 37 patients with grade $\geq 3$ cardiac AE, 18 patients (49\%) were continuing acalabrutinib at data cut-off. Six of the 37 patients (16\%) with grade $\geq 3$ cardiac $A E$ discontinued acalabrutinib due to those grade $\geq 3$ cardiac $A E$, including acute myocardial infarction $(n=2$; grade 3 and grade 5), cardiac failure congestive ( $n=2$; grade 3 and 5), cardiac failure ( $n=1$; grade 3$)$, and cardiac tamponade ( $n=1$; grade 4$)$. Thirteen of the 37 patients discontinued acalabrutinib due to other $\operatorname{AE}(n=4)$, progressive disease $(n=5)$, death $(n=3)$, and other reasons $(n=1)$. Sixteen of the 51 grade $\geq 3$ cardiac AE (31\%) led to dose delay and $36(71 \%)$ were managed with concomitant medications. In total, 43 of the grade $\geq 3$ cardiac AE (84\%) resolved, of which 15 were associated with dose delays, four resulted in drug withdrawal, and 24 had no associated dose modifications or changes. No sudden cardiac deaths were reported.

In the analysis comparing acalabrutinib monotherapy with 
Table 3. Incidence of cardiac adverse events.

\section{Event}

\section{All patients $(\mathrm{N}=762)$}

\section{Any grade}

Grade $\geq 3^{c}$

Any cardiac $\mathrm{AE},{ }^{\mathrm{a}} \mathrm{n}(\%),{ }^{\mathrm{b}}$ [number of individual events], \{rate per PEY\}

129 (17), [199], $\{0.081\}$

37 (5), [51], $\{0.023\}$

Most common cardiac AE (preferred terms; occurring in $\geq 4$ patients), $n(\%)$, [number of individual events], \{rate per PEY\}

Atrial fibrillation/flutter ${ }^{d}$

Atrial fibrillation

Atrial flutter
38 (5), [48], $\{0.024\}$

$34(4),[44],\{0.021\}$

$4(0.5),[4],\{0.003\}$
11 (1.4), [12], \{0.007\}

10 (1), [11], \{0.006\}

$1(0.1),[1],\{0.001\}$

\section{Palpitations}

Tachycardia

23 (3), [27], $\{0.014\}$

$17^{\mathrm{e}}(2),[18],\{0.011\}$

0

Sinus tachycardia

0

Angina pectoris

Bradycardia

Cardiac failure

Acute myocardial infarction

Supraventricular tachycardia

$11^{\mathrm{e}}(1),[13],\{0.007\}$

$1(0.1),[1],\{0.001\}$

2 (0.3), [2], \{0.001\}

$2(0.3),[2],\{0.001\}$

9 (1), [10], $\{0.006\}$

6 (0.8), [6], \{0.004\}

3 (0.4), [3], \{0.002\}

$5(0.7),[6],\{0.003\}$

5 (0.7), [6], \{0.003\}

$4^{\mathrm{e}}(0.5),[4],\{0.003\}$
1 (0.1), [1], \{0.001\}

${ }^{a}$ Adverse events (AE) categorized under the system organ class cardiac disorders. ${ }^{b} 199$ AE were reported in 129 patients (17\%). No events under the preferred terms sudden death or sudden cardiac death were reported. ${ }^{\circ}$ Other grade $\geq 3 \mathrm{AE}$ of interest occurring in $<4$ patients each included complete atrioventricular (AV) block ( $n=2 ; 0.3 \%)$, acute coronary syndrome $(n=1 ; 0.1 \%)$, second-degree AV block ( $n=1 ; 0.1 \%)$, and ventricular fibrillation $(n=1 ; 0.1 \%)$. dPatients with "atrial fibrillation" or "atrial flutter" preferred terms combined. There was no overlap between patients with "atrial fibrillation" and "atrial flutter" events. "One patient had both "tachycardia" and "sinus tachycardia" events. Another patient had both "sinus tachycardia" and "supraventricular tachycardia" events. A third patient had both "tachycardia" and "supraventricular tachycardia" events. All other reports of "tachycardia," "sinus tachycardia," and "supraventricular tachycardia" occurred in unique patients. PEY: patient exposure years.

the comparator arms in ASCEND and ELEVATE-TN, 45 of 333 patients (14\%) in the pooled acalabrutinib monotherapy group and 25 of 322 (8\%) in the pooled comparator group experienced cardiac AE (odds ratio, 1.86; $95 \%$ confidence interval $[\mathrm{Cl}]: 1.11-3.11 ; P=0.02)$. Grade $\geq 3$ cardiac $A E$ were reported in $4 \%(n=14)$ and $3 \%(n=10)$ of patients in the acalabrutinib and comparator groups, respectively. Atrial fibrillation was the most common cardiac AE in both groups, with incidence rates of $4 \%(n=14)$ and $2 \%(n=5)$, respectively. The proportions of patients with $\mathrm{CV}$ risk factors were similar between the pooled acalabrutinib monotherapy and pooled comparator groups among those with cardiac $A E(n=39$ [87\%] and $n=22$ [88\%], respectively) and those without cardiac $A E(n=220$ [76\%] and $n=234$ [79\%]).

\section{Atrial fibrillation/flutter adverse events}

Atrial fibrillation/flutter events of any grade were reported in 38 ( $n=15$ with treatment-naïve CLL; $n=23$ with relapsed/refractory CLL) of 762 patients (5\%). Atrial fibrillation was the most common cardiac $A E$ based on individual preferred terms and occurred in 34 patients (4\%). Median time to an event of atrial fibrillation/flutter was 17.1 months (Figure 1B) and atrial fibrillation/flutter events had a median duration of 0.1 month (range, 0.0-12.4). Rates of atrial fibrillation were consistently low over time, with approximately $1 \%(n=10 / 760)$ of acalabrutinib-treated patients having atrial fibrillation/flutter events in the first 6 months on treatment, $0.2 \%(n=1 / 653)>6$ to 12 months after treatment initiation, and $3 \%(n=16 / 585)>12$ to 24 months after starting acalabrutinib treatment. Among 38 patients with atrial fibrillation/flutter events, seven (18\%) had a prior history of arrhythmia, atrial fibrillation, or flutter. Twelve (32\%) patients with treatment-emergent atrial fibrillation/flutter were subsequently initiated on at least 1 anticoagulant due to atrial fibrillation, one of whom experienced a major hemorrhage event after starting the anticoagulant. In the full analysis population of 762 patients, 299 patients started on-study anticoagulant treatment for any reason, among whom 12 (4\%) subsequently experienced a major hemorrhage event. The major hemor- 
rhage events had resolved in nine of the 12 patients; events were ongoing in three patients at the time of data cut-off.

Twenty-nine patients had de novo atrial fibrillation/flutter (i.e., no prior history), among whom one patient experienced a subsequent stroke event (grade 3 ischemic cerebral infarction occurring 682 days after starting treatment and 2 days after the atrial fibrillation event). This patient was female and 67 years of age with a medical history of grade 2 arterial hypertension, grade 1 heart failure, and recurrent transient cerebral ischemic attacks 7 to 15 years before study entry. Notably, the patient de- veloped acute myocardial infarction on treatment day 221 and had received relevant concomitant medications prior to atrial fibrillation onset (clopidogrel, fraxiparin, aspirin). Atrial fibrillation/flutter events occurred relatively more frequently with increasing age (age <65 years: 2\% $[n=7 / 288] ; 65$ to $<75$ years: $6 \%[n=19 / 318]$; $\geq 75$ years: $8 \%$ $[n=12 / 156])$. Among patients with no history of atrial fibrillation before study enrollment $(n=706)$, the number (\%) of patients in each Shanafelt risk score category was 171 (24\%; score 0-1), 297 (42\%; score 2-3), 190 (27\%; score 4), and 48 ( $7 \%$; score $\geq 5$ ); the rate of treatment-emergent atrial fibrillation among these patients increased with in-

A

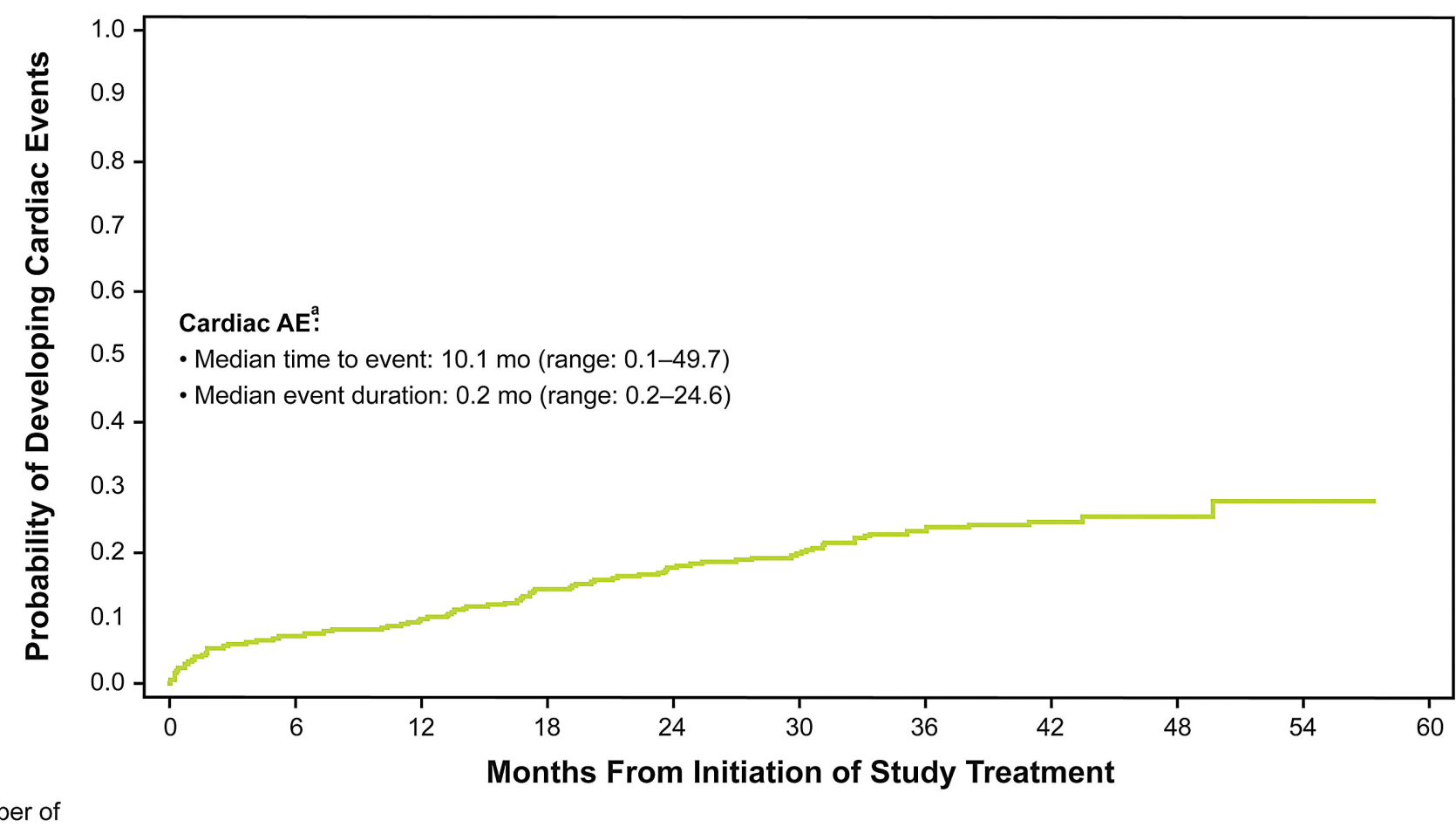

$\begin{array}{llllllllll}\text { Number of } & \\ \text { Patients at Risk } & 762 & 591 & 531 & 399 & 340 & 252 & 177 & 117 & 42\end{array}$

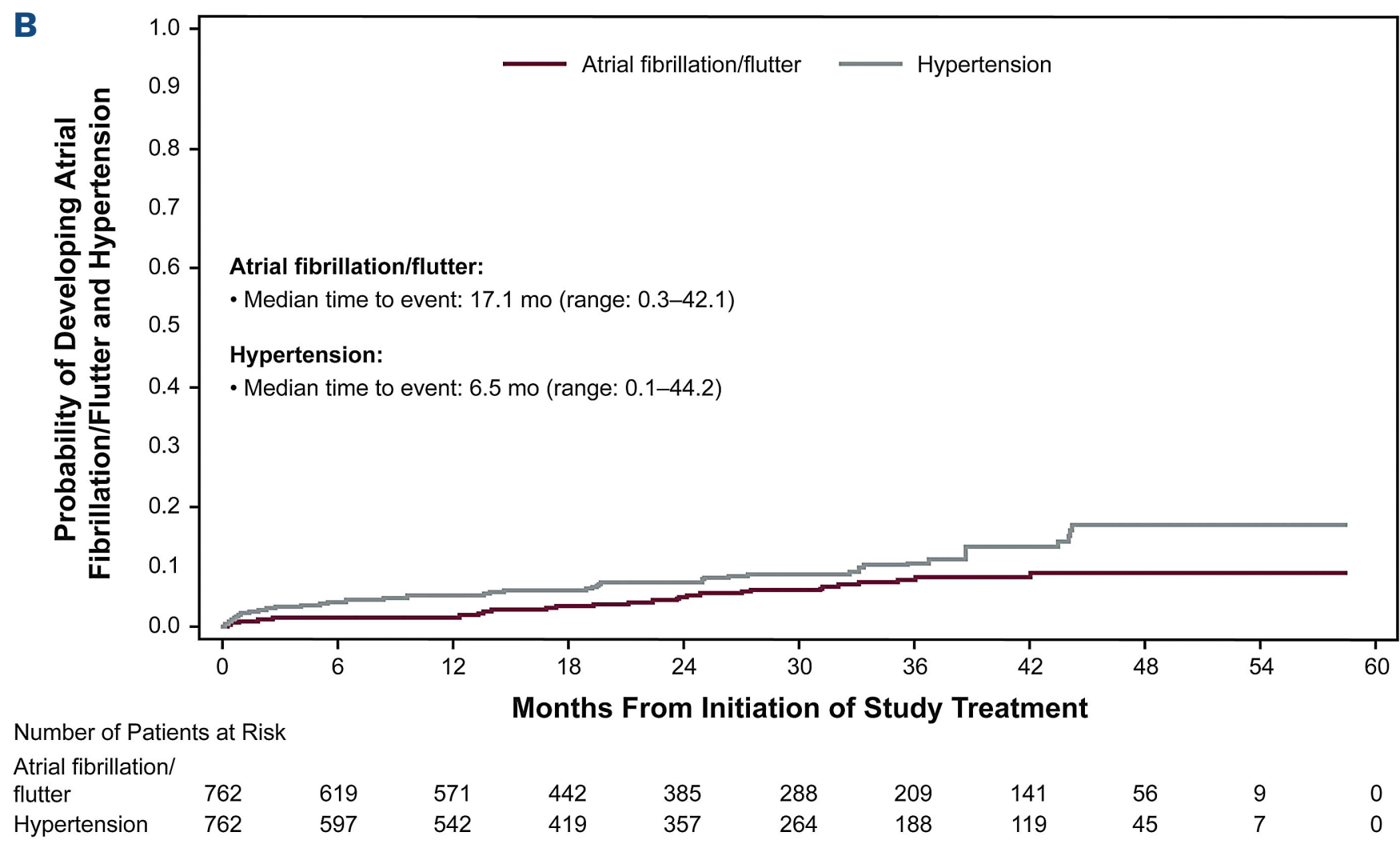

Figure 1. Time to onset of cardiac, atrial fibrillation/flutter, and hypertension adverse events. Time to onset of (A) cardiac events and (B) atrial fibrillation/flutter and hypertension adverse events (AE). AE ${ }^{a}$ categorized under the system organ class cardiac disorders; mo: months. 
creasing Shanafelt risk score category $(2 \%[n=3], 5 \%$ [n=14], 3\% [n=6], and 13\% [ $n=6]$, respectively; Online Supplementary Table S4). The cumulative incidence of atrial fibrillation events was higher in patients with Shanafelt risk scores $\geq 5$ and in those with a previous history of atrial fibrillation compared with patients with Shanafelt risks scores $\leq 4$ (Figure 2). Infections occurred concurrently in seven $(18 \%)$ of the 38 patients who experienced an atrial fibrillation/flutter event, including three patients with concurrent pneumonia.

\section{Hypertension adverse events}

In total, hypertension events were reported in 67 patients (9\%). The majority of patients with hypertension events had relapsed/refractory CLL $(n=39$ [58\%]) compared with treatment-naïve CLL $(n=28$ [42\%]). Median time to an event of hypertension was 6.5 months (Figure 1B). Patients who experienced hypertension events more commonly had risk factors for hypertension (defined in the Online Supplementary Appendix) compared with patients without hypertension events $(79 \%$ [ $n=53 / 67]$ vs. 59\% [ $n=412 / 695]$, respectively). Among the 67 patients with hypertension events, 43 (64\%) had preexisting hypertension. Other than preexisting hypertension, the most common risk factors (incidence $>5 \%$ ) included hyperlipidemia (19\% [n=13]), hypercholesterolemia (19\% [ $n=13])$, diabetes mellitus $(10 \%$ $[n=7])$, coronary artery disease $(9 \%[n=6])$, chronic obstructive pulmonary disease $(7 \%[n=5])$, and coronary ar- tery bypass (6\% [n=4]). In total, 13 of $43(30 \%)$ patients with preexisting hypertension and 0 of 24 patients without preexisting hypertension received at least one concomitant medication for hypertension management during the hypertension event.

Thirty-five grade $\geq 3$ hypertension events (grade $3, n=34$; grade $4, n=1$ ) were reported in 30 patients, among whom $24(80 \%)$ had a prior history of hypertension. Ten patients (1\%) experienced 10 grade $\geq 3$ hypertension events (29\% of total grade $\geq 3$ hypertension events) during the first 6 months of acalabrutinib treatment; $0.6 \%(n=4)$ experienced grade $\geq 3$ hypertension events $>6$ to 12 months after treatment initiation and $1 \%(n=7)>12$ to 24 months after starting treatment. Among the 30 patients with grade $\geq 3$ hypertension events, no patient discontinued acalabrutinib treatment due to these events, and 21 (70\%) were continuing acalabrutinib at data cut-off. Three of the grade $\geq 3$ hypertension AE (9\%) led to dose delay and 18 (51\%), including the three resulting in dose delay, were managed with concomitant medications. Among the 17 events that were not managed with concomitant medications or dose delay, no additional actions were documented. Two patients experienced grade 3 hypertension that required hospitalization or prolonged hospitalization; both events resolved with drug interruption/dosing delay and concomitant or additional medication. One patient had grade 4 hypertension and experienced the event while hospitalized for a grade 3 serious hypercalcemia event.

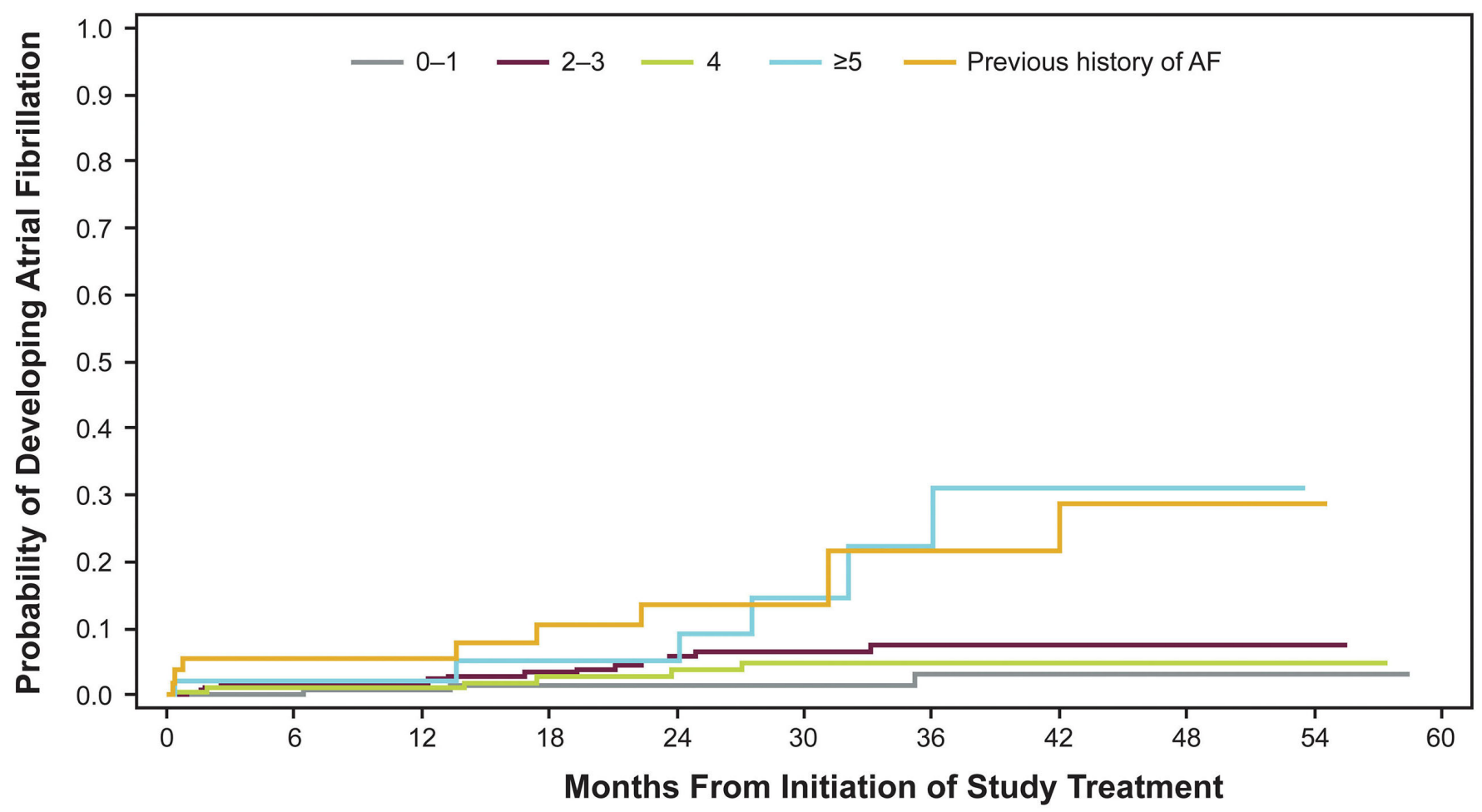

\begin{tabular}{|c|c|c|c|c|c|c|c|c|c|c|c|c|}
\hline \multicolumn{13}{|c|}{ Number of Patients at Risk } \\
\hline \multirow{4}{*}{$\begin{array}{l}\text { Shanafelt } \\
\text { risk score }\end{array}$} & $0-1$ & 171 & 142 & 132 & 99 & 85 & 74 & 60 & 41 & 13 & 3 & 0 \\
\hline & $2-3$ & 297 & 236 & 222 & 173 & 153 & 113 & 81 & 55 & 26 & 2 & 0 \\
\hline & 4 & 190 & 162 & 145 & 109 & 95 & 66 & 43 & 30 & 10 & 3 & 0 \\
\hline & $-\quad \geq 5$ & 48 & 36 & 32 & 29 & 23 & 12 & 9 & 4 & 2 & 0 & 0 \\
\hline Previous histol & of $A F$ & 56 & 43 & 40 & 32 & 29 & 23 & 16 & 11 & 5 & 1 & 0 \\
\hline
\end{tabular}

Figure 2. Time to onset of atrial fibrillation by Shanafelt risk score category and previous history of atrial fibrillation (AF). 
This patient concurrently experienced other adverse events including fall, encephalopathy, respiratory failure, and seizure and had notable medical history of severe hypertension and Guillian-Barre syndrome. In total, 20 (57\%) of the grade $\geq 3$ hypertension $A E$ resolved, including two that resulted in dose delay and 18 that had no associated dose modifications or changes; 15 (43\%) events were ongoing at data cut-off.

\section{Discussion}

In this pooled analysis of four clinical studies of acalabrutinib monotherapy in patients with CLL, the incidence of cardiac AE was relatively low. Overall, cardiac AE of any grade occurred in $17 \%$ of patients, with only a $5 \%$ incidence of atrial fibrillation/flutter events of any grade over a median 25.9 months of follow-up. Among the 129 patients who experienced cardiac AE, 91\% had preexisting cardiac risk factors. Any-grade cardiac $A E$ occurred at a consistent rate over time. While it is unknown whether some cardiac $A E$ resolve with discontinuation of drug, there were relatively few discontinuations due to cardiac $A E$ in this analysis, reported in only $1 \%$ of patients.

BTK inhibitors have become a preferred therapy in CLL; however, cardiac toxicity has been a safety concern for ibrutinib, particularly due to increased incidence of atrial fibrillation (reported incidence up to $18 \%)^{12,13,20,21,32-34}$ and sudden death. ${ }^{12,35}$ While the mechanism behind ibrutinib's cardiac toxicity profile is not well understood, a preclinical study in rats suggested ibrutinib may result in inhibition of the PI3K-Akt signaling pathway, which under normal conditions provides a cardioprotective effect on the heart under stress. ${ }^{36} \mathrm{~A}$ separate recent study in mice found that 4 weeks of ibrutinib treatment led to inducible atrial fibrillation, left atrial enlargement, myocardial fibrosis, and inflammation, while comparable treatment with acalabrutinib did not. ${ }^{37}$ Ibrutinib treatment in mice with BTKinactivating mutations still led to inducible atrial fibrillation, indicating that BTK in mice is not the relevant target. ${ }^{37}$ Chemoproteomic profiling and knockout experiments in mice suggested that C-terminal Src kinase (CSK), an off target of ibrutinib, may be responsible for ibrutinibinduced atrial fibrillation. ${ }^{37}$ While CSK inhibition was not tested directly, a biochemical and cellular profiling study of BTK inhibitors showed that acalabrutinib did not inhibit any of the Src kinases at physiologically relevant concentrations. ${ }^{11}$ A study of peripheral blood samples from patients with CLL also showed that ibrutinib inhibited kinases downstream of CSK whereas acalabrutinib did not. ${ }^{38}$ These experiments suggest that acalabrutinib would be expected to have a significantly lower rate of atrial fibrillation than ibrutinib. The relevance of these findings to humans, however, should be based on prospective studies comparing ibrutinib with acalabrutinib. ${ }^{39}$

Atrial fibrillation poses a significant burden to patients with CLL, with $6.1 \%$ of patients presenting with a history of atrial fibrillation at the time of CLL diagnosis. ${ }^{31}$ Patients with atrial fibrillation have a 5-fold increased risk of stroke and a 9-fold increased risk of developing cardiac failure. ${ }^{40-}$ 43 Management of atrial fibrillation is challenging in patients with CLL who are oftentimes older with other comorbidities such as hypertension, which also must be adequately managed to reduce the risks of stroke and bleeding in the context of anticoagulant therapy. ${ }^{40}$ Patients receiving BTK inhibitor treatment should be assessed for prior atrial fibrillation history and risk factors for stroke and bleeding in alignment with current atrial fibrillation management guidelines. ${ }^{44-46}$

It is important to note that the focus of our analysis of atrial fibrillation/flutter was on any-grade events. While grade $\geq 3$ atrial fibrillation events were observed among patients in this pooled analysis, CTCAE grading in atrial fibrillation primarily distinguishes the presence or absence of symptoms, with grades 1 and 2 representing "asymptomatic" and "non-urgent" cases of atrial fibrillation, respectively, and grades 3 and 4 representing "symptomatic" and "life-threatening" atrial fibrillation, respectively. However, evidence suggests patients with asymptomatic atrial fibrillation are at higher risk of cerebrovascular events and CV mortality than those with more typical presentation, suggesting that CTCAE grading does not adequately assess medical risk of atrial fibrillation. ${ }^{47}$ In the current analysis, atrial fibrillation/flutter of any grade was reported in $5 \%$ of patients $(n=38)$ over a median of 25.9 months of follow-up. The rate of atrial fibrillation/flutter is reasonably consistent with the incidence of new-onset atrial fibrillation after a median follow-up of 7.3 years in an untreated CLL population with no prior history of atrial fibrillation (6\%), ${ }^{31}$ and with the incidence of any-grade atrial fibrillation among placebotreated patients with asymptomatic, treatment-naïve, early stage CLL after a median follow-up of 31 months in the CLL-12 study (7\%). ${ }^{20}$ In our comparative analysis of pooled data from the ASCEND and ELEVATE-TN studies, the incidence of any-grade atrial fibrillation was $4 \%$ with acalabrutinib monotherapy compared with $2 \%$ in the pooled comparator arm. By contrast, in a previously reported analysis of the RESONATE-2 study with a similar median follow-up of 29 months, the rate of any-grade atrial fibrillation in patients with CLL receiving ibrutinib was $10 \%$ (4\% for grade $\geq 3$ atrial fibrillation), suggesting a higher risk with ibrutinib. ${ }^{48}$ In a pooled analysis of data from four randomized controlled trials of ibrutinib (median follow-up 16.6 months), approximately $63 \%$ of atrial fibrillation events reported in ibrutinib-treated patients over 24 months of treatment occurred in the first 6 months, ${ }^{13}$ whereas $29 \%$ of atrial fibrillation events in our 
analysis occurred in the first 6 months of acalabrutinib monotherapy, with a more constant rate over time. In the same pooled analysis of four ibrutinib studies, de novo atrial fibrillation events increased with increasing Shanafelt risk score category, with incidence rates ranging from $4 \%$ to $9 \%$ for categories 0 to 4 and the highest incidence of atrial fibrillation in those with Shanafelt risk score $\geq 5$ $(15 \%))^{13}$ In our analysis, the incidence of atrial fibrillation also was highest in those with the highest Shanafelt risk scores, but was generally lower across all categories compared with those reported for ibrutinib, ${ }^{13}$ with incidence rates ranging from $2 \%$ to $5 \%$ for categories 0 to 4 and $13 \%$ for categories $\geq 5$. A notable increase in the rate of atrial fibrillation was seen only in patients with the highest Shanafelt risk category, suggesting that lower risk scores may not be as informative in acalabrutinib-treated patients.

Ibrutinib treatment is associated with an increased risk of hypertension and an associated increased risk of atrial fibrillation..$^{14}$ Overall, hypertension-related events were infrequent in the present analysis (any-grade incidence of $9 \%$; grade $\geq 3$ incidence of $4 \%$ ) and only 13 of the 67 patients (19\%) with events received concurrent medication for hypertension management during the event. In addition, a majority of patients with hypertension events (64\%) had preexisting hypertension. By comparison, in a retrospective analysis of 301 patients with CLL, $71 \%$ of patients without hypertension at baseline developed new hypertension, and $56 \%$ of patients with hypertension at baseline had worsening hypertension with ibrutinib treatment. ${ }^{22}$ In a pooled analysis of four clinical trials of ibrutinib, the rates of any-grade and grade 3/4 hypertension were $10 \%$ and $4 \%$, respectively, similar to the rates reported in our study; however, the median follow-up duration was shorter in that analysis ( 16 months). ${ }^{49}$ In a separate integrated analysis of two ibrutinib clinical trials with a median treatment duration of 29 months, anygrade and grade $3 / 4$ hypertension were reported in $21 \%$ and $7 \%$ of ibrutinib-treated patients, respectively. ${ }^{50} \mathrm{Com}-$ paratively, in a phase $\mathrm{lb} / \mathrm{Il}$ study of ibrutinib in 132 patients with treatment-naïve (median follow-up, 87 months) or relapsed/refractory (median follow-up, 82 months) CLL, the incidence of grade $\geq 3$ hypertension was $28 \%$ overall, with yearly incidence rates of $9 \%$ ( $\leq 1$ year), $8 \%(>1-2$ years), 19\% (>2-3 years), 15\% (>3-4 years), 16\% ( $>4-5$ years), $16 \%$ ( $>5-6$ years), and $5 \%$ (>6-7 years). ${ }^{19}$ The results of our analysis suggest a lower risk of hypertension events with acalabrutinib, although longer follow-up is required in patients on both ibrutinib and acalabrutinib.

There are some limitations of our analysis. Although we conducted an analysis comparing pooled acalabrutinib monotherapy with the pooled comparator arms from the two phase III studies, the individual trials were not designed to detect statistical differences in AE between the treatment arms. However, baseline characteristics and risk factors were similar between the comparator groups, mitigating concerns regarding underlying differences between these patient subgroups. Furthermore, any comparisons to ibrutinib rely on cross-trial comparisons, which are fraught with confounding factors, particularly with respect to the patient population enrolled. The Shanafelt risk score used in some of our analyses is a simplified scoring system used previously in patients with CLL and therefore is included here for comparison, ${ }^{13,30,31}$ whereas guideline-recommended risk assessments for atrial fibrillation take a more comprehensive approach and may be more appropriate for use in clinical practice. ${ }^{44-46}$ In addition, our results should be considered in the context of the differing dosing schedules (though most patients were treated with $100 \mathrm{mg}$ twice daily) and treatment exposure times (0.0-58.5 months) across studies, as well as the exclusion of patients with significant CV disease. Finally, CV issues in cancer patients can take several years to manifest; longer follow-up of CV sequelae may be necessary. ${ }^{51,52}$

In conclusion, based on the results of this pooled analysis, the incidence of cardiac AE with acalabrutinib treatment is relatively low in patients with CLL. The results from our analysis also suggest an intriguing difference in atrial fibrillation and hypertension rates with acalabrutinib compared with ibrutinib. Our findings are supported by the recently reported results from the head-to-head ELEVATE-RR trial (ACE-CL-006; clinicaltrials gov. Identifier: NCT02477696), which demonstrated a statistically lower incidence of any-grade atrial fibrillation and hypertension with acalabrutinib versus ibrutinib in patients with previously treated CLL. ${ }^{39}$

\section{Disclosures}

$J R B$ has received grants or contracts from Gilead, Loxo/Lilly, Sun, TG Therapeutics, and Verastem/SecuraBio, is a consultant for AbbVie, Acerta/AstraZeneca, BeiGene, Bristol Myers Squibb/Juno/Celgene, Catapult, Dynamo, Eli Lilly, Genentech/Roche, Gilead, Kite, Loxo, MEI Pharma, Morphosys AG, Nextcea, Octapharma, Pfizer, Pharmacyclics, Rigel, Sunesis, TG Therapeutics, and Verastem, has received payment or honoraria for lectures, presentations, speakers bureaus, manuscript writing or educational events from Janssen and Teva, and has participated on data safety monitoring or advisory boards for Invectys and Morphosys. JCB is a consultant for AstraZeneca, Trillium, Syndax, Novartis, Kartos, and has ownership in Vercerx. PG has received consulting/advisory fees/honoraria from AbbVie, Acerta/AstraZeneca, Adaptive Bio, ArQule/MSD, BeiGene, Gilead, Janssen, Juno/Celgene/Bristol Myers Squibb, and Loxo/Lilly, and has received research funding from AbbVie, Gilead, Janssen, and Sunesis. JPS is an employee of the US Oncology Network, is a consultant for AbbVie, Acerta/AstraZeneca, BeiGene, Bristol Myers Squibb, Cel- 
gene, Genentech, Pharmacyclics, Pfizer, and TG Therapeutics, has ownership in VelosBio, and receives research funding from Acerta, Celgene, Genentech, Gilead, Merck, Pharmacyclics, Seattle Genetics, Takeda, and TG Therapeutics. $\mathrm{PH}$ has received travel, accommodations, and expenses from AbbVie and Janssen, research funding from AbbVie and Janssen, honoraria and research funding from F. Hoffmann-LaRoche, honoraria from AstraZeneca, and research funding from Pharmacyclics and Gilead, and is employed by the University of Leeds. DMS has participated in advisory boards for Adaptive Bio, BeiGene, Epizyme, Karyopharm, and TG Therapeutics, and has received clinical trial funding from Acerta, ArQule, Gilead, Juno, Mingsight, Novartis, Karyopharm, and Verastem. CS has received research funding from Genmab. WJ is a consultant for and currently employed by Maria Sklodowska-Curie National Research Institute of Oncology, has received research funding from and was previously employed by Jagiellonian University, and has received research funding from Janssen, Mei Pharma, Merck, Pharmacyclics, Roche, Takeda, and TG Therapeutics. JMP is a consultant for Actinium, AstraZeneca, BeiGene, Gilead, Loxo, and MEI Pharma. AF has nothing to disclose. PP is employed by and a stockholder in AstraZeneca. $L T$ is an employee of AstraZeneca. NK-C is an employee of AstraZeneca. JM is a consultant for AstraZeneca, Janssen, Bristol Myers Squibb, Boston Biomedical, Immunocure, Myovant, and Deciphera, and is supported by National Institutes of Health grants (R01HL141466, R01HL155990, and R01HL156021). RRF is a consultant for AbbVie, AstraZeneca, BeiGene, Janssen, Loxo, and Pharmacyclics, has received payment or honoraria for lectures, presentations, speakers bureaus, manuscript writing, or educational events from AbbVie, AstraZeneca, and Janssen, has received payment for expert testimony from AbbVie, and has participated on a data safety monitoring board or advisory board for Incyte.

\section{Contributions}

All authors contributed to data interpretation. Statistical and safety analyses were performed by MB and NK-C. All authors reviewed and provided important intellectual contributions to the manuscript; all authors approved the final version for publication.

\section{Acknowledgements}

The authors thank the investigators and coordinators at each of the clinical sites, and the patients who participated in the trials included in this pooled analysis and their families. This project was supported by Acerta Pharma, a member of the AstraZeneca Group. Medical writing assistance, funded by AstraZeneca, was provided by Robert J. Schoen, PharmD, and Cindy Gobbel, PhD, of Peloton Advantage, LLC, an OPEN Health company.

\section{Funding}

Support to JCB was provided by the National Cancer Institute R35 CA197734, Four Winds Foundation, and the D. Warren Brown Foundation. CS was supported by the Intramural Research Program of the National Heart, Lung, and Blood Institute.

\section{Data-sharing statement}

Data underlying the findings described in this manuscript may be obtained in accordance with AstraZeneca's data sharing policy described at https://astrazenecagrouptrials. pharmacm.com/ST/Submission/Disclosure.

\section{References}

1. National Comprehensive Cancer Network. NCCN Clinical Practice Guidelines in Oncology: Chronic Lymphocytic Leukemia/Small Lymphocytic Lymphoma version 4.2021. Available from:

https://www.nccn.org/professionals/physician_gls/default.aspx. Accessed July 20, 2021.

2. Imbruvica [package insert]. Sunnyvale, CA, Horsham, PA: Pharmacyclics, Janssen Biotech, Inc., 2020.

3. Calquence [package insert]. Wilmington, DE: AstraZeneca Pharmaceuticals, 2019.

4. Brukinsa [package insert]. San Mateo, CA: BeiGene USA, Inc, 2019.

5. Vitale C, Burger JA. Chronic lymphocytic leukemia therapy: new targeted therapies on the way. Expert Opin Pharmacother. 2016;17(8):1077-1089.

6. Pal Singh S, Dammeijer F, Hendriks RW. Role of Bruton's tyrosine kinase in $\mathrm{B}$ cells and malignancies. Mol Cancer. 2018;17(1):57.

7. Barf T, Covey T, Izumi R, et al. Acalabrutinib (ACP-196): a covalent Bruton tyrosine kinase inhibitor with a differentiated selectivity and in vivo potency profile. $J$ Pharmacol Exp Ther. 2017;363(2):240-252.

8. Tam CS, Trotman J, Opat S, et al. Phase 1 study of the selective BTK inhibitor zanubrutinib in B-cell malignancies and safety and efficacy evaluation in CLL. Blood. 2019;134(11):851-859.

9. Byrd JC, Harrington B, O'Brien S, et al. Acalabrutinib (ACP-196) in relapsed chronic lymphocytic leukemia. N Engl J Med. 2016;374(4):323-332.

10. Bond DA, Woyach JA. Targeting BTK in CLL: beyond Ibrutinib. Curr Hematol Malig Rep. 2019;14(3):197-205.

11. Kaptein A, de Bruin G, Emmelot-van Hoek M, et al. Potency and selectivity of BTK inhibitors in clinical development for B-cell malignancies. Blood. 2018;132(Supplement 1):1871.

12. Salem JE, Manouchehri A, Bretagne M, et al. Cardiovascular toxicities associated with ibrutinib. J Am Coll Cardiol. 2019;74(13):1667-1678.

13. Brown JR, Moslehi J, O'Brien S, et al. Characterization of atrial fibrillation adverse events reported in ibrutinib randomized controlled registration trials. Haematologica. 2017;102(10):17961805. 
14. Dickerson T, Wiczer T, Waller A, et al. Hypertension and incident cardiovascular events following ibrutinib initiation. Blood. 2019;134(22):1919-1928.

15. Caldeira D, Alves D, Costa J, Ferreira JJ, Pinto FJ. Ibrutinib increases the risk of hypertension and atrial fibrillation: systematic review and meta-analysis. PLoS One. 2019;14(2):e0211228.

16. Byrd JC, Brown JR, O'Brien S, et al. Ibrutinib versus ofatumumab in previously treated chronic lymphoid leukemia. $\mathrm{N}$ Engl J Med. 2014;371(3):213-223.

17. Byrd JC, Hillmen P, O'Brien S, et al. Long-term follow-up of the RESONATE phase 3 trial of ibrutinib vs ofatumumab. Blood. 2019;133(19):2031-2042.

18. Burger JA, Tedeschi A, Barr PM, et al. Ibrutinib as initial therapy for patients with chronic lymphocytic leukemia. N Engl J Med. 2015;373(25):2425-2437.

19. Byrd JC, Furman RR, Coutre SE, et al. Ibrutinib treatment for first-line and relapsed/refractory chronic lymphocytic leukemia: final analysis of the pivotal phase 1b/2 PCYC-1102 study. Clin Cancer Res. 2020;26(15):3918-3927.

20. Langerbeins P, Bahlo J, Rhein C, et al. Ibrutinib versus placebo in patients with asymptomatic, treatment-naïve early stage CLL: primary endpoint results of the phase 3 double-blind randomized CLL12 trial. Hematol Oncol. 2019;37(suppl 2):38-40.

21. Ganatra S, Sharma A, Shah S, et al. Ibrutinib-associated atrial fibrillation. JACC Clin Electrophysiol. 2018;4(12):1491-1500.

22. Jones J, George BS, Peterson CB, et al. Hypertension, cardiovascular and renal complications observed in patients with chronic lymphocytic leukemia receiving long-term therapy with ibrutinib. Blood. 2020;136(Supplement 1):27-28.

23. Sharman JP, Egyd M, Jurczak W, et al. Acalabrutinib with or without obinutuzumab versus chlorambucil and obinutuzmab for treatment-naive chronic lymphocytic leukaemia (ELEVATE TN): a randomised, controlled, phase 3 trial. Lancet. 2020;395(10232):1278-1291.

24. Ghia P, Pluta A, Wach M, et al. ASCEND: Phase III, randomized trial of acalabrutinib versus idelalisib plus rituximab or bendamustine plus rituximab in relapsed or refractory chronic lymphocytic leukemia. J Clin Oncol. 2020;38(25):2849-2861.

25. Byrd JC, Furman RR, Coutre SE, et al. Targeting BTK with ibrutinib in relapsed chronic lymphocytic leukemia. N Engl J Med. 2013;369(1):32-42.

26. Byrd JC, Wierda WG, Schuh A, et al. Acalabrutinib monotherapy in patients with relapsed/refractory chronic lymphocytic leukemia: updated phase 2 results. Blood. 2020;135(15):12041213.

27. Awan FT, Schuh A, Brown JR, et al. Acalabrutinib monotherapy in patients with chronic lymphocytic leukemia who are intolerant to ibrutinib. Blood Adv. 2019;3(9):1553-1562.

28. Byrd JC, Woyach JA, Furman RR, et al. Acalabrutinib in treatment-naive (TN) chronic lymphocytic leukemia (CLL): updated results from the phase 1/2 ACE-CL-001 study. Blood. 2018; 132(Suppl 1):S692.

29. Sun CCL, Nierman PK, Kendall EK, et al. Clinical and biological implications of target occupancy in CLL treated with the BTK inhibitor acalabrutinib. Blood. 2020;136(1):93-105.

30. Archibald WJ, Rabe KG, Kabat BF, et al. Atrial fibrillation in patients with chronic lymphocytic leukemia (CLL) treated with ibrutinib: risk prediction, management, and clinical outcomes. Ann Hematol. 2021;100(1):143-155.

31. Shanafelt TD, Parikh SA, Noseworthy PA, et al. Atrial fibrillation in patients with chronic lymphocytic leukemia (CLL). Leuk Lymphoma. 2017;58(7):1630-1639.

32. Farooqui M, Valdez J, Soto S, Bray A, Tian X, Wiestner A. Atrial fibrillation in CLL/SLL patients on ibrutinib. Blood. 2015;126(23):2933.

33. Burger JA, Barr PM, Robak T, et al. Long-term efficacy and safety of first-line ibrutinib treatment for patients with CLL/SLL: 5 years of follow-up from the phase 3 RESONATE-2 study. Leukemia. 2020;34(3):787-798.

34. Moslehi JJ. Cardiovascular toxic effects of targeted cancer therapies. N Engl J Med. 2016;375(15):1457-1467.

35. Lampson BL, Yu L, Glynn RJ, et al. Ventricular arrhythmias and sudden death in patients taking ibrutinib. Blood. 2017;129(18):2581-2584.

36. McMullen JR, Boey EJ, Ooi JY, Seymour JF, Keating MJ, Tam CS. Ibrutinib increases the risk of atrial fibrillation, potentially through inhibition of cardiac PI3K-Akt signaling. Blood. 2014;124(25):3829-3830.

37. Xiao L, Salem JE, Clauss S, et al. Ibrutinib-mediated atrial fibrillation attributable to inhibition of C-terminal Src kinase. Circulation. 2020;142(25):2443-2455.

38. Patel V, Balakrishnan K, Bibikova E, et al. Comparison of acalabrutinib, a selective Bruton tyrosine kinase inhibitor, with ibrutinib in chronic lymphocytic leukemia cells. Clin Cancer Res. 2017;23(14):3734-3743.

39. Byrd JC, Hillmen P, Ghia P, et al. Acalabrutinib versus ibrutinib in previously treated chronic lymphocytic leukemia: results of the first randomized phase 3 trial. J Clin Oncol. $2021 \mathrm{Jul} 26$. 2021;39(31):3441-3452.

40. Win NT, Teo SP. Atrial fibrillation in older patients-reducing stroke risk is not only about anticoagulation. J Geriatr Cardiol. 2016;13(10):880-882.

41. Wolf PA, Abbott RD, Kannel WB. Atrial fibrillation as an independent risk factor for stroke: the Framingham Study. Stroke. 1991;22(8):983-988.

42. Verma A, Kalman JM, Callans DJ. Treatment of patients with atrial fibrillation and heart failure with reduced ejection fraction. Circulation. 2017;135(16):1547-1563.

43. Chatterjee NA, Chae CU, Kim E, et al. Modifiable risk factors for incident heart failure in atrial fibrillation. JACC Heart Fail. 2017;5(8):552-560.

44. January CT, Wann LS, Calkins H, et al. 2019 AHA/ACC/HRS focused update of the 2014 AHA/ACC/HRS Guideline for the Management of Patients With Atrial Fibrillation: a report of the American College of Cardiology/American Heart Association Task Force on Clinical Practice Guidelines and the Heart Rhythm Society in collaboration with the Society of Thoracic Surgeons. Circulation. 2019;140(2):e125-e151.

45. January CT, Wann LS, Alpert JS, et al. 2014 AHA/ACC/HRS guideline for the management of patients with atrial fibrillation: executive summary: a report of the American College of Cardiology/American Heart Association Task Force on Practice Guidelines and the Heart Rhythm Society. Circulation. 2014;130(23):2071-2104.

46. Hindricks G, Potpara T, Dagres N, et al. 2020 ESC Guidelines for the diagnosis and management of atrial fibrillation developed in collaboration with the European Association for Cardio-Thoracic Surgery (EACTS): the Task Force for the diagnosis and management of atrial fibrillation of the European Society of Cardiology (ESC) Developed with the special contribution of the European Heart Rhythm Association (EHRA) of the ESC. Eur Heart J. 2021;42(5):373-498.

47. Siontis KC, Gersh BJ, Killian JM, et al. Typical, atypical, and asymptomatic presentations of new-onset atrial fibrillation in the community: Characteristics and prognostic implications. Heart Rhythm. 2016;13(7):1418-1424.

48. Barr PM, Robak T, Owen C, et al. Sustained efficacy and detailed 
clinical follow-up of first-line ibrutinib treatment in older patients with chronic lymphocytic leukemia: extended phase 3 results from RESONATE-2. Haematologica. 2018;103(9):15021510.

49. O'Brien S, Hillmen P, Coutre S, et al. Safety analysis of four randomized controlled studies of ibrutinib in patients with chronic lymphocytic leukemia/small lymphocytic lymphoma or mantle cell lymphoma. Clin Lymphoma Myeloma Leuk. 2018;18(10):648-657.
50. Coutre SE, Byrd JC, Hillmen P, et al. Long-term safety of singleagent ibrutinib in patients with chronic lymphocytic leukemia in 3 pivotal studies. Blood Adv. 2019;3(12):1799-1807.

51. Moslehi J. The cardiovascular perils of cancer survivorship. $\mathrm{N}$ Engl J Med. 2013;368(11):1055-1056.

52. Groarke JD, Cheng S, Moslehi J. Cancer-drug discovery and cardiovascular surveillance. N Engl J Med. 2013;369(19):17791781. 